\title{
A survey of service Discovery Architecture of MANET with AODV-SD
}

\author{
Bela I. Patel \\ Institute of Science and \\ Technology for Advanced \\ Studies and Research \\ Vallabh Vidhyanagar, \\ India
}

\begin{abstract}
Mobile Adhoc Network (MANET) is network of a no. of mobile routers and associated hosts, organized in a random fashion via wireless link. MANET has been widely used for not only military purposes but for search-and- rescue operations, intelligent transportation system, data collection, virtual classrooms and ubiquitous computing. There are various Issues in MANET like Routing, MAC Layer issues, Transport protocol, QoS, Data Mgt. And Security etc. from them Service Discovery is one of the most important issues in MANET. Service discovery technologies are exploited to enable services to advertise their existence in a dynamic way, and can be discovered, configured and used by other devices with a minimum of manual efforts. Most of the service discovery protocols such as DEAPspace, UPnP, Konark, Salutation, Jini, and SLP. These protocols don't provide an appropriate route from consumer to service provider. Hence after services are discovered, a route request needs to be initiated in order to access the service. In this paper proposing an efficient, robust and flexible approach to service discovery for MANET that not only discovers a service provider, but at the same time, it also provides a route to access the service.
\end{abstract}

Keywords: Mobile Ad hoc Networks, Service Discovery and Service, Discovery Protocols, Jini, UPnP, Konark, SLP, Salutation.

\section{INTRODUCTION}

A wireless ad hoc network is a collection of autonomous nodes or terminals that communicate with each other by forming a multichip radio network and maintaining connectivity in a decentralized manner. Since the nodes communicate over wireless links, they have to contend with the effects of radio communication, such as noise, fading, and interference.

In MANET service discovery is most important issue. A service can be anything that can be useful for someone. For example when a labourer works for cleaning a house, he is giving his services for which he is paid. Similarly a professor teaches to his students providing them knowledge that is useful for students. This act of teaching is a service provided by a professor to his students. So, service can be defined as any facility provided by a device that can be useful for any other device. A service could be a software service like providing an implementation of some algorithm (for example, converting one audio file format to another) on a device so that when some device needs this service, it can contact that device and use it. A service can also be a hardware service like a printer that can be used by a mobile device to print a file. To get benefit from these services a device must be able to locate them in the network and also have the ability to invoke these services. Service Discovery Protocols (SDPs) provide such capabilities to devices.

In this paper, proposing an efficient, robust and flexible approach to service discovery in mobile ad-hoc network. First, we will provide a review of existing approaches of service discovery architecture with its advantages and disadvantages. We will then discuss our proposing approach to service discovery. Finally we will conclude the paper with future work.

\section{LITERATURE REVIEW}

Service Discovery Protocols can be defined as "Service Discovery Protocol is $\mathrm{n} / \mathrm{w}$ protocols which allow automatic detection of devices and services offered by these devices on a computer $\mathrm{n} / \mathrm{w}$.' The service discovery protocols proposed in paper that is classified as;

- Directory less architecture (DEAPspace, UPnP, Konark).

- Directory based architecture.

"A directory is an entity that stores information about services available in the network so as to enable service discovery and invocation."

The directory based architecture can be divided into two categories:

a. Centralized directory architecture (Salutation, Jini, and SLP)

"Relies on one or a few centralized directories that store the descriptions of all services available in the network."

b. Distributed directory architecture.

"Directories are further distributed and deployed dynamically."

1. infrastructure-less distributed directory architecture

2. Infrastructure-based distributed directory architecture.

\section{JINI Architecture}

Basic Jini Functions

$\checkmark \quad$ Allows a service (hardware, software) to make itself known to the community.

$\checkmark \quad$ Allows clients (browsers, OSs, hardware) within a community to discover services of interest. 
$\checkmark \quad$ Network Communication between peers.

$\checkmark \quad$ Knowledge of the network terrain.

$\checkmark$ Finding services.

$\checkmark \quad$ Utilizing services.

Advantages

$\checkmark \quad$ Scalable Implementation of architecture.

$\checkmark \quad$ OS and hardware independence.

$\checkmark$ Scalable Scope (local to internet URL).

$\checkmark$ Requires more limited apriority knowledge.

$\checkmark \quad$ Jini provides a more flexible and robust service discovery infrastructure for building distributed components comparing to other systems.

$\checkmark \quad$ The Jini object code offers direct access to the service using an interface known to the client.

$\checkmark$ This code mobility replaces the necessity of preinstalling drivers on the client.

$\checkmark \quad$ The Jini specifications are open source and may be used freely.

$\checkmark \quad$ The code mobility appears as an efficient solution for supporting information retrieval.

$\checkmark$ The Jini discovery infrastructure supports both unicast and multicast service discovery protocol.

$\checkmark$ Jini systems provide mechanisms for service construction, lookup, communication, and use in a distributed system.

$\checkmark$ Examples of services include devices such as printers, displays, or disks; software such as applications or utilities;

Disadvantages

$\checkmark$ The problem is that the code mobility is very complex in an environment which bandwidth is a scarce resource and users' mobility makes continuous communication.

$\checkmark \quad$ Since code mobility gives to the users the access to other machines, security is a concern and the literature hardly addresses the security of MANET.

$\checkmark \quad$ The nodes should be designed to define in such a way that the peers accept the others' code.

$\checkmark$ Since code mobility gives to the users the access to other machines, security is a concern, and the literature hardly addresses the security of Ad Hoc networks.

$\checkmark \quad$ Bleeding edge technology, not supported by industry yet

$\checkmark \quad$ Network Appliance market is very much embedded hardware based, and Java is not yet.

\section{UPNP Architecture}

Actions in UPNP (Universal Plug and Play)

$\checkmark \quad$ Discover devices \& services

$\checkmark$ Get a description of the device

$\checkmark$ Control discovered devices

$\checkmark$ Be informed of events indicating changes in the device

$\checkmark \quad$ Use a presentation prepared by the device to present a control

Advantages

$\checkmark$ Universal Plug and Play is the youngest of these protocols, and it is still in an early state of development.

$\checkmark \quad$ Microsoft plans to implement it for all Windows platforms.

$\checkmark \quad$ The specifications and a sample source code are available freely. $\checkmark$ It is processor, operating system and communication protocol independent.

Disadvantages

$\checkmark$ UPnP is designed for TCP/IP networks only.

$\checkmark \quad$ In its current version, it does not allow clients to search for service attributes.

\section{SALUTATION Architecture}

Salutation architecture is composed of two major components:

- $\quad$ Salutation Manager(SLM)

The Salutation Manager is the core of the architecture, similar to the Lookup Service in Jini.

- Transport Manager.

Provides reliable communication channels, regardless of the underlying network transport.

SLM can be discovered by services in a number of ways such as:

$\checkmark \quad$ Using a static table that stores the transport address of the remote SLM.

$\checkmark$ Sending a broadcast discovery query using the protocol defined by the Salutation architecture.

$\checkmark$ Inquiring the transport address of a remote SLM through a central directory server. This protocol is undefined by the Salutation architecture; however, the current specification suggests the use of SLP.

$\checkmark$ The service specifies the transport address of a remote SLM directly.

Advantages

$\checkmark$ Salutation is independent on the network technology and may run over multiple infrastructures, such as over TCP/IP and IrDA.

$\checkmark \quad$ It is not limited to HTTP over UDP over IP, as $\mathrm{UPnP}$ is

$\checkmark$ Moreover, Salutation is independent on the programming language, i.e., it is not limited to nor does it have a prerequisite for Java (as Jini).

$\checkmark \quad$ Its major advantage compared to UPnP and Jini is that there already exist commercial implementations.

$\checkmark$ A Salutation Manager sits on the Transport Managers that provide reliable Communication channels, regardless of what the underlying network transports are.

$\checkmark$ The Salutation Manager provides a transportindependent interface to Server and Client applications.

$\checkmark$ Salutation can operate in any network, including IP, IR and, in the future, wireless. This transport independence is the strongest feature of Salutation.

Disadvantages

$\checkmark \quad$ The Salutation is well defined but confined to the service discovery protocol and session management.

$\checkmark \quad$ Salutation accordingly doesn't address features like remote event notification, which are no doubt useful in distributed environment.

SLP Architecture

SLP (service location Protocols) offers the following services:

$\checkmark$ Obtaining service handles for User Agents.

$\checkmark \quad$ Maintaining the directory of advertised services. 
$\checkmark$ Discovering available service attributes.

$\checkmark$ Discovering available Directory Agents.

$\checkmark$ Discovering the available types of Service Agents.

Advantages

$\checkmark \quad$ SLP offers a flexible and scalable architecture and the utilization of service templates make service browsing and human interaction possible.

$\checkmark$ Since SLP is able to operate with or without a DA, it is suitable for networks of different sizes, ranging from very small ad hoc connectivity to large enterprise networks.

$\checkmark \quad$ SLP also includes a leasing concept with a lifetime that defines how long a DA will store a service registration.

$\checkmark \quad$ SLP also can conduct in the distributed scenario without DAs, where UAs repeatedly multicast Attribute Request to network and receive the unicast replies from SAs directly.

$\checkmark \quad$ This mode provides a relative simple structure for service discovery in small network (such as home LAN).

$\checkmark$ SLP is developed by an open and vendor independent forum and its implementation is freely available. We expect SLP to play a major role in service discovery.

$\checkmark \quad$ SLP is independent on the programming language.

$\checkmark \quad$ The SLP also supports a simple service registration leasing mechanism that handles the cases where service hardware is broken but the services continue to be advertised

Disadvantages

$\checkmark \quad$ Tends to increase the bandwidth usage

\subsection{Existing Approach Vs Proposed Approach}

After analyzing the Existing service discovery protocols it can be concluded that The Existing service discovery protocols don't provide an appropriate route from consumer to service provider. Hence, after services are discovered, a route request needs to be initiated in order to access the service. AODV, Totally different approach of service Discovery, Provided a service Discovery at network layer. As a result when a service discovery request is initiated to discover a service, a route is also established towards the service provider. Hence, when the client wants to use the service, a new route request is not required. It offers "quick adaptation to dynamic link conditions, low processing and memory overhead, low network utilization, Determines unicast routes to destinations within the ad -hoc network".

\section{OVERVIEW OF AODV-SD}

We have extended The AODV protocol to perform service discovery; we call this extension AODV-SD.RREQ, RREP, RERR. Three message types are defined by AODV. In order to perform service discovery with AODV, we have extended the formats of the RREQ and RREP messages. Every node will be periodically broadcasting the services provided by it to its peer nodes

\subsection{Service Discovery with AODV}

New fields were defined, but also actions that nodes along the network perform when receiving these extended messages. whenever a node requires a service, it performs a lookup in its service table, services table maintains a information about services provided by current node as well as information about services provided by other nodes, The current node services information itself when the node in initialized, or when new service is initialized within the node while the other node services information is acquired when the current node participates in service discovery process. Each row in a services table contains the service identifier (a string that uniquely identifies the service), its IP address, a lifetime is used to keep information up to date, and a list of attributes that varies with the type of service and URL path. (Figure 1).

\begin{tabular}{|c|c|c|c|c|c|c|}
\hline Service & P & Protocol & IP & Lifetime & Attributes & URL \\
ID & o & & Address & & List & Path \\
& & & & & & \\
\hline & & & & & \\
\hline
\end{tabular}

Figure. 1 Services Table

A node can contact a service provider to use service, if the service information in its service table has a valid route to the service provider, and if the service lifetime is still valid; otherwise node has to initiate a discovery process by sending an RREQ message with extensions, known as SREQ. Recall that these extensions are fields added to regular RREQ, so it is possible to the locate the service (IP address of the service provider), as well as a route to it, simultaneously. RREQ message extension includes a Type field to identify a SREQ message, the Length of the extension, the Service ID field Length, Attributes List field length, service ID and attribute list with an optional values used to include restrictions in the useful service. (Figure 2).

\begin{tabular}{l|c|c|c|}
\hline 1 byte & 1 byte & 1 byte & 1 byte \\
\hline & & & \\
\hline Type & Length & $\begin{array}{c}\text { Length } \\
\text { Service ID }\end{array}$ & $\begin{array}{c}\text { Length } \\
\text { Attributes List }\end{array}$ \\
\hline \multicolumn{4}{|c|}{ Service ID } \\
\hline \multicolumn{4}{|c|}{ Atributes List } \\
\hline
\end{tabular}

Fig 2. Format of SREQ messages

When a node receives an SREQ it executes several following actions. First, it determines a valid association (Service name, IP address) for the required service is already available in its services table; that means if it provides the service or if it knows a valid route to a node that can provide it. If particular information is found, the node issues an RREP message with extensions for service discovery, also known as SREP, and sends it to the requesting node. SREQ and SREQ messages are transmitted through the network according to the rules at the AODV protocol, so both service provider routes will be found, as well as the reverse route, will be found. the RREP message extension includes the following fields: a Type to identify SREP messages, the extensions Length, lifetime of service, the service URL Length, and finally the URL itself (Figure 3). 


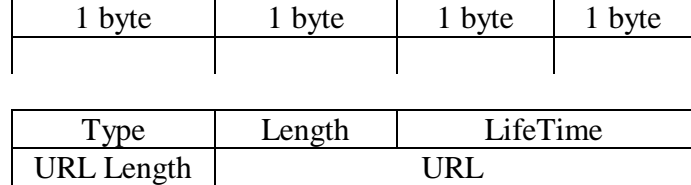

Fig 3 . Format of SREP messages

If a node contains an association (service name, IP address) to a service, but does not have a valid route to it, then it assigns this IP address as the address to resolve issuing an SREQ. Any node receiving an SREQ with a valid destination address sends an SREP if it has a route to the destination node or if it knows an equivalent route to the service required. Otherwise, if it does not have information about the service required, or a route to the destination node, it will only forward the SREQ message. If a node receives an SREP and already has information about the service required, it compares the lifetime with the lifetime contained in its service table; if the information in the table is more recent, then it discards the message received and issues an SREP with that information. Otherwise, it will simply send the message to its destination.

\section{SIMULATION PARAMETERS}

We adopted the LBNL network simulator (ns) to evaluate the effects of performance of ad hoc routing protocols. The ns is a very popular software for simulating advanced TCP/IP algorithms and wireless ad hoc networks. To simulate our approach, we have developed our own simulation software. The simulator creates $\mathrm{n}$ nodes dynamically and links them randomly. Every node is randomly chosen to be either capable of storing or not storing advertisements. Nodes are randomly assigned a number of services.

\section{CONCLUSION}

In this paper we explored several architecture choices for service discovery with its advantages and disadvantages from them the proposed AODV approach is very flexible, efficient and can be adopted to work in any environment. In addition to it not only pull the service provider information on demand, but a node will also be pushing the service advertisements periodically along with route information.

\section{FUTURE WORK}

We can improve the current work to represent the service using better representation language like DAML etc. In addition, broadcasting of service advertisement right now done periodically. We can improve the broadcasting mechanism.

\section{REFERENCES}

[1] Dante Arias Torres, J. Antonio Garcia-Macias, "Service Discovery in Mobile Ad-hoc Networks by Extending the AODV Protocol", Proc. 2nd Mobile Computing Workshop (ENC' 04). Colima, Mexico. Sept. 2004. ISBN 970-692-170-2

[2] A Novel Approach to Service Discovery in Mobile Adhoc Network by Noman Islam Zubair A. Shaikh

[3] "Understanding Universal Plug and Play", White Paper, UPnP Forum (http://www.upnp.org), June 2000.

[4] Cho, C. and Lee, D., "Survey of Service Discovery Architectures for Mobile Ad hoc Networks", Term paper, Mobile Computing, CEN 5531, Department of Computer and Information Science and Engineering (CICE), University of Florida, Fall, 2005.
[5] Samba Sesay, Zongkai Yang and Jianhua He, "A Survey on Mobile Ad Hoc Wireless Network", Information Technology Journal 3 (2): 168-175, 2004, ISSN 16826027, (c) 2004 Asian Network for Scientific Information.

[6] "JiniTm Architecture Specification", Version 1.0.1, November 1999

[7] Service Location Protocol, "RFC 2608", http://www.ietforg, June 1999.

[8] Michael Nidd, "Reducing Power Use in DEAPspace Service Discovery", IBM Research Zurich Research Laboratory, 2000.

[9] Fatma Outay, Veronique and Ridha Bouallegue, "Survey of Service Discovery Prtocols and Benefits of Comining Service and Route Discovery", International Journal of Computer Science and Network Security, VOL. 7 No 11, November, 2007.

[10] Choonhwa Lee and Sumi Helal, "Protocols For Service Discovery In Dynamic And Mobile Networks", International Journal of Computer Research, Volume 11, Number 1, pp. 1-12, (C) 2002 Nova Science Publishers,Inc.

[11] Survey of Service Discovery Protocols in Mobile Ad Hoc Networks by Adnan Noor Mian, Roberto Beraldi, Roberto Baldoni. 